\title{
Optimal and Robust Distributed Resource Allocation for SWIPT-Enabled Cognitive Networks
}

\author{
Yongjun Xu, Member, IEEE, Haijian Sun, Member, IEEE, Jie Yang, Member, IEEE, \\ Guan Gui, Senior Member, IEEE, and Song Guo, Fellow, IEEE
}

\begin{abstract}
Simultaneous wireless information and power transfer (SWIPT)-enabled cognitive networks (CRNs) is recognized as one of the most promising techniques to improve spectrum efficiency and prolong operation lifetime in 5G and beyond. However, existing methods focus on the centralized algorithm and the power allocation under perfect channel state information (CSI). The analytical solution and the impact of power splitting (PS) on the optimal power allocation strategy are not addressed. In addition, the influence of the PS factor on the feasible region of transit power is rarely analyzed. In this paper, we propose a joint power allocation and PS algorithm under perfect CSI and imperfect CSI, respectively, for multiuser SWIPT-enabled CRNs scenarios. The power minimization of resource allocation problem is formulated as a multivariate nonconvex optimization which is hard to obtain the closed-form solution. Hence, we propose a suboptimal algorithm to alternatively optimize the power allocation and PS coefficient under the cases of the lowharvested energy region and the high-harvested energy region, respectively. Moreover, a closed-form distributed power allocation and PS expressions are derived by the Lagrangian approach. Simulation results confirm the proposed method with good robustness and high energy efficiency.
\end{abstract}

Index Terms-Cognitive networks, SWIPT, resource allocation, distributed algorithm.

\section{INTRODUCTION}

With the increasing number of mobile terminals, energy consumption becomes a huge problem. How to prolong the network lifetime and reduce power consumption have attracted lots of intention from both industry and academia. Currently, simultaneous wireless information and power transfer (SWIPT) is proposed for extending the life cycle

This work was supported by the Project Funded by the National Science and Technology Major Project of China under Grant TC190A3WZ-2 the National Natural Science Foundation of China under Grant 61671253 the Jiangsu Specially Appointed Professor under Grant RK002STP16001, the Innovation and Entrepreneurship of Jiangsu High-level Talent under Grant CZ0010617002, the Six Top Talents Program of Jiangsu under Gran XYDXX-010, the 1311 Talent Plan of Nanjing University of Posts and Telecommunications. (Corresponding author: Guan Gui)

$\mathrm{Y}$. Xu is with the School of Communication and Information Engineering, Chongqing University of Posts and Telecommunications, Chongqing 400065 China. Y. Xu is also with the Shandong Provincial Key Lab of Wireles Communication Technologies, Shandong University, Jinan 250100, China. (Email: xuyj@cqupt.edu.cn)

H. Sun is with the Department of Computer Science, University of Wisconsin-Whitewater, Whitewater, WI 53190, USA. (E-mail: h.j.sun@ieee.org)

J. Yang and G. Gui are with with the College of Telecommunications and Information Engineering, Nanjing University of Posts and Telecommunications, Nanjing 210003, China. (E-mail: \{jyang, guiguan\}@njupt.edu.cn)

S. Guo is with Department of Computing, The Hong Kong Polytechnic University, Hung Hom, Kowloon, Hong Kong (E-mail: song.guo@ polyu.edu.hk). of energy-limited communication networks by harvesting surrounding radio frequency [1]-[4]. According to the policy of the SWIPT, wireless information is divided into two kinds of signals: energy-harvesting signal and information-decoding signal. Specifically, the former can allow wireless terminals to harvest the radio frequency (RF) energy so that it is a good method to provide the sustainable power. The latter can support information decoding for the basic quality of service (QoS) of users. As a result, the works on SWIPT technique have been concerned by many scholars [5]-[7].

On the other side, cognitive radio (CR) is proposed to improve spectrum efficiency (SE) by permitting secondary users (SUs) to access the spectrum owned by primary users (PUs) [8]-[12]. Nevertheless, in practical communication environments, system performance may be strongly constrained by the energy-constrained users, e.g., wireless sensor networks (WSNs), device-to-device (D2D) networks. Therefore, applying the SWIPT technique in CR networks (CRNs) can not only guarantee good SE but also improve the lifetime of networks by harvesting the surrounding RF energy. As a result, SWIPTenabled CRNs have become an effective technique for the next-generation communication network.

SWIPT-enabled CRNs face greater connatural challenges than the conventional CRNs and traditional SWIPT networks [13]. On the one hand, resource allocation (RA) problems become more complex due to the introducing multivariate parameters (e.g., transmit power and power splitting (PS) ratio), which can lead to a nonconvex optimization problem. On the other hand, the energy harvesting $(\mathrm{EH})$ technique may bring some nonlinear characteristics to the conventional system, which cause excessive data processing and message overhead. Thus, in the view of computational complexity, the low-cost totally distributed RA algorithm is more significant in SWIPT-enabled CRNs.

The RA problem has been widely investigated in SWIPTenabled CRNs from the aspect of power optimization, beamforming optimization, time splitting optimization, etc. In [14], the joint delay balance and power allocation problem was studied to achieve the energy efficiency (EE) maximization of SUs for an EH CRNs with a stochastic Stackelberg game approach. The authors in [15] investigated the joint power control and time allocation problem to achieve the sum-rate maximization of SUs for wireless powered CRNs, where SUs first harvest energy in the downlink wireless power transfer phase and then use the energy for data transmission in the uplink wireless information transmission phase in a timedivision way. In [16], the optimal RA for the harvested energy 
maximization of SUs was studied for a wideband CRN with SWIPT. In [17], a simultaneous cooperative spectrum sensing and $\mathrm{EH}$ model was proposed to improve the transmission performance of the multichannel $\mathrm{CRN}$, where the aggregate throughput and EE were maximized by jointing optimizing the sensing time and transmission power. In [18], the optimal RA problem for the harvested energy maximization of PUs was studied for wireless power transfer in CRNs by using the support vector machine and the particle swarm optimization technique. In [19], the bandwidth utility maximization problem with optimal time slot allocation was formulated for achieving the proportional fairness transmission in a cognitive wireless powered communication network. In [20], the sum throughput maximization of SUs was studied for a wireless powered CRN with a non-linear EH model. The RA problem for secure communication was studied for cooperative cognitive wireless powered communication networks [21]. However, the above works are studied under the assumption of perfect channel state information (CSI).

In practical communication systems, it is difficult to obtain the exact channel parameters due to the randomness of wireless channels. Moreover, the inherent non-linearities of practical EH hardware may cause the estimation errors. Thus, robust RA problems are very important for practical cognitive SWIPT networks. In [22], under the worst-case channel uncertainty model, the robust transceiver design for SWIPT in multiple-input-multiple-output (MIMO) underlay CRNs was investigated to maximize the sum harvested power at the energy harvesting receivers meanwhile guaranteeing the required minimum mean-square-error (MMSE) at the secondary information-decoding (ID) receiver and the interference constraints at the primary receivers. But the PS factor optimization was not neglected. In [23], the multi-objective optimization problem with the aim of jointly minimizing the total transmit power and maximizing the EH efficiency was investigated for multiple-input-single-output (MISO) CRNs with SWIPT where the CSI of the link between the secondary transmitter and idle secondary receiver was modeled by using the bounded channel uncertainty set. To guarantee secure communication and $\mathrm{EH}$, in [24], the problem of robust secure artificial noise-aided beamforming and power splitting design was investigated for a MISO CRN with SWIPT under the bounded CSI error model and the probabilistic CSI error. In [25], the robust max-min fairness RA in sensing-based wideband CRN with SWIPT was studied under the assumption of imperfect spectrum sensing and CSI. In [26], to balance the consumed power and the harvested power, a robust RA problem was studied in a MISO CRN with SWIPT under imperfect CSI.

\section{A. Motivation and Contributions}

Although in the above-mentioned literature, CSI is generally assumed to be imperfect, the PS design is rarely involved. The PS factor is the key metric for balancing the optimality of information transmission and the harvested energy, which needs to be designed carefully. Moreover, the low-cost distributed algorithm can reduce overhead among different users but is not addressed. Furthermore, the influence of the PS factor on the feasible region of available transmission power is rarely analyzed, which is also important to reduce the complexity of the algorithm by relaxing some strong constraints.

In this paper, the optimal and robust RA problem is investigated for a multiuser SWIPT-enabled cognitive ad hoc network, where multiple pairs of SUs can use the licensed spectrum owned by one pair of PUs in an underlay spectrum sharing way and the PS-based protocol is used at the EH receiver. The contributions are summarized as follows.

- A total power minimization RA problem (i.e., this objective leads to a low total interference power leakage in general) is formulated subject to the constraint on transmit power, harvested energy, interference temperature, user's signal-to-interference-plus-noise ratio (SINR) and PS coefficient. The transmit power and PS design are jointly optimized to reduce the energy consumption for prolonging the lifetime of wireless networks.

- The relationship between the user's SINR and the harvested energy is analyzed to reflect the impact of PS coefficient on the available transmit power. Under perfect CSI, the nonconvex problem is firstly decomposed into the problem with the low-harvested energy and the one with the high-harvested energy, which are transformed into the convex problem and resolved with closed-form solutions. Under spherical channel uncertainties, the robust RA problem is converted into a convex one by using the worst-case approach. The robust sensitivity is also deduced.

- We further propose a distributed RA algorithm for the ease of information exchange among different users. Compared with centralized algorithm which requires more dedicated computation resources and overhead during communication, our algorithm is more suitable for low-power and small devices such as Internet of Things (IoTs).

\section{B. Paper Organization}

The rest of this paper is organized as follows. Section II introduces the system model and problem formulation. Section III presents the transformation process and feasible region analysis of the system model. Section IV gives the optimal RA with perfect CSI under low-energy level and high-energy level. The robust RA with imperfect CSI is given in Section V. Section VI presents the simulation results. Conclusions are introduced in Section VII. The abbreviations used in this paper are summarized in Table I.

\section{SySTEM MODEL AND FORMULATION}

Consider a cognitive ad hoc network with SWIPT where each receiver has equipped the SWIPT function. There are $M$ SU links (e.g., $\forall i, m \in \mathcal{M}=\{1,2, \cdots, M\}$ ) and one pair of PUs. There is no central control base station (BS) and each receiver can harvest the electromagnetic energy from the surrounding environment to prolong the lifetime of each node. The PS-based protocol [2] is applied in this model. Specifically, the received signal at the receiver is processed by 
TABLE I

ABBREVIATIONS.

\begin{tabular}{l|l}
\hline Abb. & Definition \\
\hline AWGN & additive white gaussian noise \\
\hline BS & base station \\
\hline CR & cognitive radio \\
\hline CRN & cognitive radio network \\
\hline CSI & channel state information \\
\hline D2D & device-to-device \\
\hline EE & energy efficiency \\
\hline EH & energy harvesting \\
\hline ID & information-decoding \\
\hline IoTs & Internet of Things \\
\hline KKT & Karush-Kuhn-Tucker \\
\hline MIMO & multiple-input-multiple-output \\
\hline MISO & multiple-input-single-output \\
\hline MMSE & minimum mean-square-error \\
\hline PU & primary user \\
\hline PU-Rx & PU receiver \\
\hline PU-Tx & PU transmitter \\
\hline PS & power splitting \\
\hline QoS & quality of service \\
\hline RA & resource allocation \\
\hline RF & radio frequency \\
\hline SE & spectrum efficiency \\
\hline SU & secondary user \\
\hline SU-Tx & SU transmitter \\
\hline SU-Rx & SU receiver \\
\hline SINR & signal-to-interference-plus-noise ratio \\
\hline SWIPT & simultaneous wireless information and power transfer \\
\hline WSN & wireless sensor network \\
\hline & \\
\hline & \\
\hline SU
\end{tabular}

a power splitter: a portion of the received power is split for $\mathrm{EH}$ and the other energy is split for ID [27]. That is to say that the receiver can achieve $\mathrm{EH}$ and information processing simultaneously. The considered model can be envisioned in the fifth generation communication networks, such as D2D network, wireless sensor networks, where energy-limited devices exist in the communication scenario. We assume that all SUs can share the same spectrum with PUs and each user has a single antenna. The SUs can estimates the channel gain from SU transmitter (SU-Tx) to each PU receiver (PU-Rx) by listening to the feedback signals from the PU-Rx and broadcast the channel information to the other SUs for transmission power control [28], [29]. The symbol definition used in the paper is summarized in Table II.

Since the sampled additive white gaussian noise (AWGN) of RF band to the baseband signal conversion is much smaller than the sum interference power from PUs and SUs, therefore, the noise from energy harvesting can be assumed to be zero [25], [30]. The interference from the PU transmitter (PU-Tx) to each SU receiver (SU-Rx) is assumed to be included in the noise [13]. Based on these assumptions, the received SINR at the $m$-th SU-Rx can be expressed as

$$
\gamma_{m}=\frac{\left(1-\rho_{m}\right) p_{m} h_{m, m}}{\left(1-\rho_{m}\right) \sum_{i \neq m}^{M} p_{i} h_{i, m}+\sigma_{m}} .
$$

Based on the linear energy harvesting model [31], the power of the received signal for the energy harvesting at the SU-Rx
TABLE II

SYSTEM PARAMETERS

of link $m$ can be expressed as

$$
E_{m}=\theta \sum_{i=1}^{M} \rho_{m} p_{i} h_{i, m}
$$

To maintain the lifetime of secondary network, the considered RA problem with total power minimization is

$$
\begin{gathered}
\min _{\left\{p_{m}, \rho_{m}\right\}} \sum_{m=1}^{M} p_{m} \\
\text { s.t.C } C_{1}: \sum_{m=1}^{M} p_{m} g_{m} \leq I^{t h}, \\
C_{2}: \gamma_{m} \geq \gamma_{m}^{\text {min }}, \\
C_{3}: E_{m} \geq E_{m}^{\text {min }}, \\
C_{4}: p_{m} \leq p_{m}^{\text {max }}, \\
C_{5}: 0 \leq \rho_{m} \leq 1,
\end{gathered}
$$

where $C_{1}$ and $C_{2}$ can satisfy the QoS guarantee of the PU and each SU, respectively. $C_{3}$ denotes the minimum harvested energy requirement for maintaining the lifetime of each SU. $C_{4}$ is constrained by the battery capacity.

Since there is no channel uncertainty in problem (3), it is a nominal optimization problem (i.e., non-robust problem) under perfect CSI [?], [32]. In general, problem (3) is nonconvex due to the coupled variables $p_{m}, \rho_{m}, \forall m$ in $C_{2}$ and $C_{3}$. Thus it is difficult to directly obtain the closed-form solutions. We have the following Lemma 1.

Lemma 1: If the harvested energy threshold satisfies $E_{m}^{m i n} \leq \frac{\theta \rho_{m} \gamma_{m}^{m i n} \sigma_{m}}{1-\rho_{m}}$, the SINR constraint can be simplified as $p_{m} h_{m, m} \geq \frac{\gamma_{m}^{m i n} \sigma_{m}}{1-\rho_{m}}$. Otherwise, it can be simplified the linear constraint $p_{m} h_{m, m} \geq \frac{\gamma_{m}^{\min }}{1+\gamma_{m}^{\min }}\left(\frac{\sigma_{m}}{1-\rho_{m}}+\frac{E_{m}^{\text {min }}}{\theta \rho_{m}}\right)$. The above discussions can be summarized as follows

$$
p_{m} h_{m, m} \geq \bar{H}_{m}=\left\{\begin{array}{l}
\frac{\gamma_{m}^{m i n} \sigma_{m}}{1-\rho_{m}}, E_{m}^{\text {min }} \leq \frac{\theta \rho_{m} \gamma_{m}^{m i n} \sigma_{m}}{1-\rho_{m}}, \\
H_{m}, \text { otherwise }
\end{array}\right.
$$

where $H_{m}=\frac{\gamma_{m}^{\min }}{1+\gamma_{m}^{\min }}\left(\frac{\sigma_{m}}{1-\rho_{m}}+\frac{E_{m}^{\min }}{\theta \rho_{m}}\right)$. The proof is provided in Appendix A.

Additionally, when $0 \leq \rho_{m} \leq 0.5, \frac{\rho_{m}}{1-\rho_{m}} \leq 1$ holds. We 
can simplify (4) as

$$
p_{m} h_{m, m} \geq \bar{H}_{m}=\left\{\begin{array}{l}
\frac{\gamma_{m}^{m i n} \sigma_{m}}{1-\rho_{m}}, E_{m}^{m i n} \leq \theta \gamma_{m}^{m i n} \sigma_{m} \\
H_{m}, \text { otherwise }
\end{array}\right.
$$

Remark 1: when $\rho_{m} \leq 0.5$ holds, it means that the EH efficiency is lower than that of the ID for data transmission. The system mainly focuses on information transmission. Otherwise, the system tends to harvest more energy to store for supporting the long-time operation or uplink transmission. As a result, compared with $C_{2}$, the Eq. (4) is decoupled with respect to the transmit power $p_{m}$, which is easier for optimal power design.

Based on (3) and (4), we have the following equivalent optimization problem

$$
\begin{gathered}
\min _{\left\{p_{m}, \rho_{m}\right\}} \sum_{m=1}^{M} p_{m} \\
\text { s.t. } C_{1}, C_{3}, C_{4}, C_{5}, \\
\bar{C}_{2}: p_{m} h_{m, m} \geq \bar{H}_{m} .
\end{gathered}
$$

\section{TRANSFORMATION AND FEASIBILITY ANALYSIS}

Though easier compared with its original form in (3), problem (6) is still difficult to solve due to the coupled variables $p_{m}, \rho_{m}$. If we want to simplify the solution process and reduce the complexity of power allocation, it is better to combine the constraints $\bar{C}_{2}$ and $C_{3}$ into one constraint, which is helpful to obtain the analytical solution of power allocation and the PS coefficient.

\section{A. Transformation of System Model}

Case 1: When $E_{m}^{m i n} \leq \frac{\theta \rho_{m} \gamma_{m}^{m i n} \sigma_{m}}{1-\rho_{m}}$, the SINR constraint of each SU (e.g., $\bar{C}_{2}$ ) can be rewritten as

$$
p_{m} h_{m, m} \geq \frac{\gamma_{m}^{\min } \sigma_{m}}{1-\rho_{m}} .
$$

According to (2), the constraint $C_{3}$ can be rewritten as

$$
p_{m} h_{m, m} \geq \frac{E_{m}^{m i n}}{\theta \rho_{m}}-Z_{m},
$$

where $Z_{m}=\sum_{i \neq m}^{M} p_{i} h_{i, m} \geq 0$. According to $E_{m}^{m i n} \leq$ $\frac{\theta \rho_{m} \gamma_{m}^{\min } \sigma_{m}}{1-\rho_{m}}$, we have the following relationship

$$
\begin{aligned}
& f^{1}=\frac{E_{m}^{m i n}}{\theta \rho_{m}}-Z_{m} \leq \frac{\theta \rho_{m} \gamma_{m}^{m i n} \sigma_{m}}{1-\rho_{m}} \times \frac{1}{\theta \rho_{m}}-Z_{m} \\
& =\frac{\gamma_{m}^{m i n} \sigma_{m}}{1-\rho_{m}}-Z_{m} .
\end{aligned}
$$

Therefore,

$$
\max f^{1}=\frac{\gamma_{m}^{\min } \sigma_{m}}{1-\rho_{m}}-Z_{m} \leq \frac{\gamma_{m}^{\min } \sigma_{m}}{1-\rho_{m}} .
$$

Combining (7), (8) with (10), we only have one constraint (7). The problem (6) becomes the following problem with the
TABLE III

FEASIBLE REGION ANALYSIS.

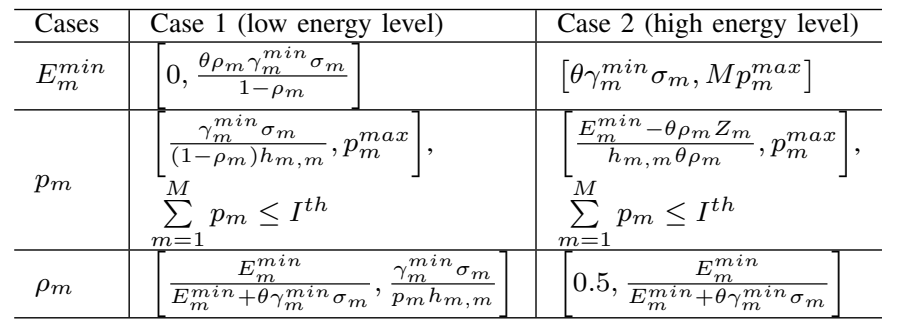

low-harvested energy requirement

(P1)

$$
\begin{gathered}
\min _{\left\{p_{m}, \rho_{m}\right\}} \sum_{m=1}^{M} p_{m} \\
\text { s.t. } C_{1}, C_{4}, C_{5}, \\
\bar{C}_{2}: p_{m} h_{m, m} \geq \frac{\gamma_{m}^{m i n} \sigma_{m}}{1-\rho_{m}}, \\
C_{6}: E_{m}^{\text {min }} \leq \frac{\theta \rho_{m} \gamma_{m}^{m i n} \sigma_{m}}{1-\rho_{m}} .
\end{gathered}
$$

Case 2: When $E_{m}^{\text {min }} \geq \frac{\theta \rho_{m} \gamma_{m}^{m i n} \sigma_{m}}{1-\rho_{m}}$, the SINR constraint of each SU (e.g., $\bar{C}_{2}$ ) becomes

$$
p_{m} h_{m, m} \geq H_{m} .
$$

Since the harvested energy threshold $E_{m}^{\min }$ is a bounded value, thus we have

$$
\frac{\theta \rho_{m} \gamma_{m}^{\min } \sigma_{m}}{1-\rho_{m}}<E_{m}^{\min } \leq E_{m}^{\max } .
$$

Ignore the impact of PU and consider an ideal case, the maximum harvesting energy is $E_{m}^{\max }=M p_{m}^{\max }$ according to (2).

Lemma 2: Under $E_{m}^{\text {min }} \geq \frac{\theta \rho_{m} \gamma_{m}^{m i n} \sigma_{m}}{1-\rho_{m}}$, we have the following relationship

$$
\frac{E_{m}^{\min }}{\theta \rho_{m}}-Z_{m} \geq H_{m}
$$

The proof is given in Appendix B.

Thus, the EH constraint $C_{3}$ and the SINR constraint $\bar{C}_{2}$ can be integrated into the EH constraint, which means the SINR constraint of each FU $C_{2}$ can be replaced by $p_{m} h_{m, m} \geq$ $\frac{E_{m}^{m i n}}{\theta \rho_{m}}-Z_{m}$. Since $E_{m}=\theta \rho_{m}\left(p_{m} h_{m, m}+Z_{m}\right) \geq E_{m}^{m i n}$ and $E_{m}^{m i n} \geq \frac{\theta \rho_{m} \gamma_{m}^{m i n} \sigma_{m}}{1-\rho_{m}}$, we have $E_{m} \geq \frac{\theta \rho_{m} \gamma_{m}^{m i n} \sigma_{m}}{1-\rho_{m}}$. And the optimization problem (7) becomes the following problem with the high-harvested energy requirement

(P2)

$$
\begin{aligned}
& \min _{\left\{p_{m}, \rho_{m}\right\}} \sum_{m=1}^{M} p_{m} \\
& \text { s.t. } C_{1}, C_{4}, C_{5}, \\
& \bar{C}_{6}: E_{m} \geq \frac{\theta \rho_{m} \gamma_{m}^{m i n} \sigma_{m}}{1-\rho_{m}} .
\end{aligned}
$$

\section{B. Feasible Region Analysis}

Case 1: feasible region analysis of (P1). From the constraint $C_{5}$ and $C_{6}$, it is easy to obtain the available PS ratio $\frac{1}{1+\theta \gamma_{m}^{m i n} \sigma_{m} / E_{m}^{m i n}} \leq \rho_{m} \leq 1$. Based on the constraint $\bar{C}_{2}$, we have $\rho_{m} \leq 1-\frac{\gamma_{m}^{m i n} \sigma_{m}}{p_{m} h_{m}}$. Since $\frac{\gamma_{m}^{m i n} \sigma_{m}}{p_{m} h_{m}}>0$, there must 
be $\left(1-\frac{\gamma_{m}^{\min } \sigma_{m}}{p_{m} h_{m}}\right)<1$. There, the PS ratio need to satisfy the interval $\frac{1}{1+\theta \gamma_{m}^{\text {min }} \sigma_{m} / E_{m}^{\text {min }}} \leq \rho_{m} \leq \frac{\gamma_{m}^{\text {min }} \sigma_{m}}{p_{m} h_{m}}$. Based on the constraint $\bar{C}_{2}$, we can derive the low bound of transmit power $p_{m}^{m i n}=\frac{\gamma_{m}^{m i n} \sigma_{m}}{\left(1-\rho_{m}\right) h_{m, m}}$. Thus, combining with $C_{4}$ for any user $m$, the transmit power should be satisfied from the interval $\frac{\gamma_{m}^{\min } \sigma_{m}}{\left(1-\rho_{m}\right) h_{m, m}} \leq p_{m} \leq p_{m}^{\max }$. Moreover, since $p_{m} \leq \frac{\gamma_{m}^{\min } \sigma_{m}}{\rho_{m} h_{m}}$ and $p_{m} \geq \frac{\gamma_{m}^{m i n} \sigma_{m}}{\left(1-\rho_{m}\right) h_{m}}$, we have $\frac{\gamma_{m}^{m i n} \sigma_{m}}{\left(1-\rho_{m}\right) h_{m}} \leq \frac{\gamma_{m}^{m i n} \sigma_{m}}{\rho_{m} h_{m}}$. Thus we have $\rho_{m} \leq 0.5$. If any fading channel gain satisfies $g_{m} \leq 1$ [33], we have $\sum_{m=1}^{M} p_{m} g_{m} \leq \sum_{m=1}^{M} p_{m} \leq I^{t h}$.

Case 2: feasible region analysis of (P2). Similarly, according to the constraint $C_{5}$ and $\bar{C}_{6}$, the PS ratio is determined by the interval $0.5 \leq \rho_{m} \leq \frac{1}{1+\theta \gamma_{m}^{m i n} \sigma_{m} / E_{m}^{m i n}}$. Since $0.5 \leq \rho_{m}$, thus we have $\frac{\rho_{m}}{1-\rho_{m}} \geq 1$. And we have the relationship $E_{m}^{\min } \geq \theta \gamma_{m}^{\min } \sigma_{m}$. Based on (13), we have the worst-case energy harvesting threshold $\theta \gamma_{m}^{\text {min }} \sigma_{m} \leq E_{m}^{\text {min }} \leq M p_{m}^{\max }$. Additionally, based on the analysis above (15), we have

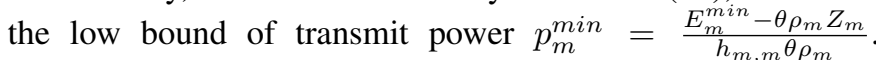
Combining with $C_{4}$, we have the range of transmit power $\frac{E_{m}^{m i n}-\theta \rho_{m} Z_{m}}{h_{m, m} \theta \rho_{m}} \leq p_{m} \leq p_{m}^{\max }$ and $\sum_{m=1}^{M} p_{m} \leq I^{t h}$. The analysis result is summarized in Table III.

\section{Optimal RA with Perfect CSI}

Although the objective is a linear combination which is convex [34], the problems (P1) and (P2) are difficult to obtain the globally optimal solutions due to the coupled variables $p_{m}, \rho_{m}$. In this section, we propose the suboptimal solutions by decomposing them into two subproblems under the fixed PS coefficient and the optimal transmit power, which is commonly used to the scenarios of the multi-objective optimization [24], [25], [35].

\section{A. Optimal RA under Low-Energy Level}

1) Power Allocation with the Fixed PS Ratio: Under the fixed PS coefficient, (P1) can be reformulated as

$$
\begin{aligned}
& \text { (P1.1) } \min _{p_{m}} \sum_{m=1}^{M} p_{m} \\
& \text { s.t. } C_{1}, C_{4}, \\
& \bar{C}_{2}: \frac{1}{p_{m} h_{m, m}} \leq \frac{1-\rho_{m}}{\gamma_{m}^{m i n} \sigma_{m}} .
\end{aligned}
$$

It is a convex optimization problem due to the linear constraints [35]. Thus, in order to obtain the closed-form solutions, the Lagrangian dual decomposition method can be used to solve this problem. The Lagrangian function of the problem (16) can be rewritten by

$$
\begin{aligned}
& L^{p}\left(\left\{p_{m}\right\}, \lambda,\left\{\beta_{m}\right\},\left\{\alpha_{m}\right\}\right)=\sum_{m=1}^{M} p_{m} \\
& +\lambda\left(\sum_{m=1}^{M} p_{m} g_{m}-I^{t h}\right)+\sum_{m=1}^{M} \beta_{m}\left(p_{m}-p_{m}^{\max }\right) \\
& \quad+\sum_{m=1}^{M} \alpha_{m}\left(\frac{1}{p_{m} h_{m, m}}-\frac{1-\rho_{m}}{\gamma_{m}^{\text {min }} \sigma_{m}}\right)
\end{aligned}
$$

where $\lambda \geq 0, \beta_{m} \geq 0$, and $\alpha_{m} \geq 0$ are the Lagrange multipliers corresponding to the power constraints. The function (17) can be rewritten as

$$
\begin{aligned}
& L^{p}\left(\left\{p_{m}\right\}, \lambda,\left\{\beta_{m}\right\},\left\{\alpha_{m}\right\}\right)=\sum_{m=1}^{M} L_{m}^{p}\left(\left\{p_{m}\right\}, \lambda,\left\{\beta_{m}\right\},\left\{\alpha_{m}\right\}\right) \\
& -\lambda I^{t h}-\sum_{m=1}^{M} \beta_{m} p_{m}^{\text {max }}-\sum_{m=1}^{M} \alpha_{m} \frac{1-\rho_{m}}{\gamma_{m}^{m i n} \sigma_{m}}
\end{aligned}
$$

where

$L_{m}^{p}\left(\left\{p_{m}\right\}, \lambda,\left\{\beta_{m}\right\},\left\{\alpha_{m}\right\}\right)=p_{m}+\lambda p_{m} g_{m}+\beta_{m} p_{m}+\frac{\alpha_{m}}{p_{m} h_{m, m}}$.

The dual problem is

$$
\begin{aligned}
& \max _{\lambda, \beta_{m}, \alpha_{m}} D\left(\lambda,\left\{\beta_{m}\right\},\left\{\alpha_{m}\right\}\right) \\
& \text { s.t. } \lambda \geq 0, \beta_{m} \geq 0, \alpha_{m} \geq 0,
\end{aligned}
$$

where the dual function is

$$
D\left(\lambda,\left\{\beta_{m}\right\},\left\{\alpha_{m}\right\}\right)=\min _{p_{m}} L^{p}\left(\left\{p_{m}\right\}, \lambda,\left\{\beta_{m}\right\},\left\{\alpha_{m}\right\}\right) .
$$

From (20) and (21), it is clear to know that the dual problem is decomposed into two layers. The inner layer optimization problem is to obtain the optimal power allocation $p_{m}$. Then the outer layer problem is to solve the dual variables (or called Lagrange multipliers).

According to (19), the Lagrangian dual function can be decomposed into $M$ subproblems for each user. According to the Karush-Kuhn-Tucker (KKT) conditions, the optimal power allocation is calculated by $\frac{\partial L_{m}^{p}(\cdot)}{\partial p_{m}}=0$, i.e.,

$$
p_{m}^{*}=\sqrt{\frac{\alpha_{m}}{h_{m, m}\left(1+\lambda g_{m}+\beta_{m}\right)}} .
$$

Additionally, the outer layer optimization problem can be solved by the subgradient method, the Lagrange multipliers can be updated as

$$
\begin{gathered}
\lambda(t+1)=\left[\lambda(t)+\xi_{1}(t) \times\left(\sum_{m=1}^{M} p_{m} g_{m}-I^{t h}\right)\right]^{+}, \\
\beta_{m}(t+1)=\left[\beta_{m}(t)+\xi_{2}(t) \times\left(p_{m}-p_{m}^{\max }\right)\right]^{+}, \\
\alpha_{m}(t+1)=\left[\alpha_{m}(t)+\xi_{3}(t) \times\left(\frac{1}{p_{m} h_{m}}-\frac{1-\rho_{m}}{\gamma_{m}^{\min } \sigma_{m}}\right)\right]^{+},
\end{gathered}
$$

where $[x]^{+}=\max (0, x) . t$ is the iteration index. $\xi_{1}(t), \xi_{2}(t)$, and $\xi_{3}(t)$ are positive step sizes at iteration $t$. From (22)-(25), it is a distributed power allocation algorithm based on local information.

2) PS Design with the Fixed Transmit Power: Under the optimal power $p_{m}$, (P1) can be reformulated as the following 
PS optimization problem (i.e., convex problem), i.e.,

$$
\begin{gathered}
\text { (P1.2) } \min _{\rho_{m}} \sum_{m=1}^{M} p_{m} \\
\text { s.t. } C_{5}, \bar{C}_{2}: \rho_{m} \leq 1+\frac{\gamma_{m}^{\text {min }} \sigma_{m}}{p_{m} h_{m}}, \\
\tilde{C}_{6}: \frac{1}{\rho_{m}} \leq 1+\frac{\theta \gamma_{m}^{m i n} \sigma_{m}}{E_{m}^{\text {min }}} .
\end{gathered}
$$

Since $\frac{\gamma_{m}^{m i n} \sigma_{m}}{p_{m} h_{m}}>0$, the problem (26) can be rewritten as

$$
\begin{aligned}
& \min _{\rho_{m}} \sum_{m=1}^{M} p_{m} \\
& \text { s.t. } C_{5}, \tilde{C}_{6} .
\end{aligned}
$$

Therefore, the Lagrangian function of the problem (27) is

$$
\begin{aligned}
& L^{p s}\left(\left\{\rho_{m}\right\},\left\{\lambda_{m}^{p s}\right\},\left\{\beta_{m}^{p s}\right\}\right)=\sum_{m=1}^{M} p_{m}+\sum_{m=1}^{M} \lambda_{m}^{p s}\left(\rho_{m}-1\right) \\
& +\sum_{m=1}^{M} \beta_{m}^{p s}\left(\frac{1}{\rho_{m}}-\left(1+\frac{\theta \gamma_{m}^{\text {min }} \sigma_{m}}{E_{m}^{\text {min }}}\right)\right) \\
& =\sum_{m=1}^{M} L_{m}^{p s}\left(\left\{\rho_{m}\right\},\left\{\lambda_{m}^{p s}\right\},\left\{\beta_{m}^{p s}\right\}\right)-\sum_{m=1}^{M} \lambda_{m}^{p s} \\
& -\sum_{m=1}^{M} \beta_{m}^{p s}\left(1+\frac{\theta \gamma_{m}^{\min } \sigma_{m}}{E_{m}^{\text {min }}}\right)+\sum_{m=1}^{M} p_{m},
\end{aligned}
$$

where $\lambda_{m}^{p s} \geq 0$ and $\beta_{m}^{p s} \geq 0$ are the Lagrange multipliers. $L_{m}^{p s}\left(\left\{\rho_{m}\right\},\left\{\lambda_{m}^{p s}\right\},\left\{\beta_{m}^{p s}\right\}\right)=\lambda_{m}^{p s} \rho_{m}+\beta_{m}^{p s} \frac{1}{\rho_{m}}$. Similarly, we have the closed-form PS coefficient, i.e.,

$$
\rho_{m}^{*}=\sqrt{\beta_{m}^{p s} / \lambda_{m}^{p s}}
$$

Obviously, it is also a distributed PS algorithm based on the local information. The Lagrange multipliers in (29) can be updated by the same approach.

\section{B. Optimal RA under High-energy Level}

1) Power Allocation with the Fixed PS Ratio: Under the fixed PS coefficient, (P2) can be reformulated as

$$
\begin{aligned}
\text { (P2.1) } & \min _{p_{m}} \sum_{m=1}^{M} p_{m} \\
\text { s.t. } & C_{1}, C_{4}, \\
\bar{C}_{6} & : \frac{1}{p_{m} h_{m, m}} \leq \frac{1-\rho_{m}}{\gamma_{m}^{\text {min }} \sigma_{m}-\left(1-\rho_{m}\right) Z_{m}} .
\end{aligned}
$$

Since (30) is a convex problem with the variable $p_{m}$, it can be solved by the same approach mentioned above.

Therefore, the optimal power is

$$
p_{m}^{*}=\sqrt{\frac{\alpha_{m}^{h}}{h_{m, m}\left(1+\lambda^{h} g_{m}+\beta_{m}^{h}\right)}} .
$$

And the Lagrange multipliers are updated by

$$
\begin{gathered}
\lambda^{h}(t+1)=\left[\lambda^{h}(t)+\xi_{6}(t) \times\left(\sum_{m=1}^{M} p_{m} g_{m}-I^{t h}\right)\right]^{+}, \\
\beta_{m}^{h}(t+1)=\left[\beta_{m}^{h}(t)+\xi_{7}(t) \times\left(p_{m}-p_{m}^{\max }\right)\right]^{+}, \\
\alpha_{m}^{h}(t+1)=\left[\alpha_{m}^{h}(t)+\xi_{8}(t) \times\left(\frac{1}{p_{m} h_{m, m}}\right.\right. \\
\left.\left.-\frac{1-\rho_{m}}{\gamma_{m}^{\text {min } \sigma_{m}}-\left(1-\rho_{m}\right) Z_{m}}\right)\right]^{+},
\end{gathered}
$$

where $\xi_{6}(t), \xi_{7}(t)$, and $\xi_{8}(t)$ are positive step sizes.

2) PS Design with the Fixed Transmit Power: Under the optimal power $p_{m}$, (P2) can be reformulated as the following PS optimization problem (i.e., convex problem), i.e.,

$$
\begin{gathered}
\text { (P2.2) } \min _{\rho_{m}} \sum_{m=1}^{M} p_{m} \\
\text { s.t. } C_{5}, \bar{C}_{6},
\end{gathered}
$$

Based on the Lagrange dual approach, (35) can be efficiently solved. Similarly, we have the closed-form PS coefficient, i.e.,

$$
\rho_{m}^{*}=\max \left(0,1-\sqrt{\beta_{m}^{p s} / \lambda_{m}^{p s}}\right),
$$

where

$$
\begin{gathered}
\lambda_{m}^{h p s}(t+1)=\left[\lambda_{m}^{h p s}(t)+\xi_{9}(t) \times\left(\rho_{m}-1\right)\right]^{+}, \\
\beta_{m}^{h p s}(t+1)=\left[\beta_{m}^{h p s}(t)+\xi_{10}(t) \times\left(\frac{1}{1-\rho_{m}}-\frac{p_{m} h_{m, m}+Z_{m}}{\gamma_{m}^{m i n} \sigma_{m}}\right)\right]^{+},
\end{gathered}
$$

where $\xi_{9}(t)$ and $\xi_{10}(t)$ are positive step sizes. Thus an iterative algorithm (Algorithm 1) is designed to obtain the optimal power allocation and PS ratio.

Remark 2: In the considered SWIPT-enabled cognitive ad hoc networks, there is no central BS for scheduling the overall messages. Based on the broadcast channel gain $g_{m}$ (which is obtained by listening to the feeback signals from the PU-Rx [28]) and the estimated interference power at each SU-Rx, the algorithm can be achieved by a distributed way from (22), (29), (31) and (36). Specifically, algorithm 1 is a distributed RA algorithm which can reduce the computational burden of mobile devices and save energy. Moreover, it can reduce the overhead of message passing among all SUs. Specifically, firstly, according to (22), (29), (31), and (36), the utility function of each SU $(\forall m)$ only depends on that user's primal variables. Secondly, the Lagrange multipliers can be divided into the local variables for each SU (e.g., $\beta_{m}, \alpha_{m}$ ).

\section{ROBUST RA WITH IMPERFECT CSI}

Due to the nature of the random channel and estimation errors in the radio environment, we cannot ignore the impact of channel uncertainties in the design of RA algorithms [32].

\section{A. Uncertainty Model}

Since there is one pair of PU in the system, assume the effect of interference uncertainty from the PBS to SU-Rx is small. Therefore, the PU's interference can be exactly obtained. The interference uncertainty of PU is not discussed in this part. Moreover, the interference to the PU-Rx and the 


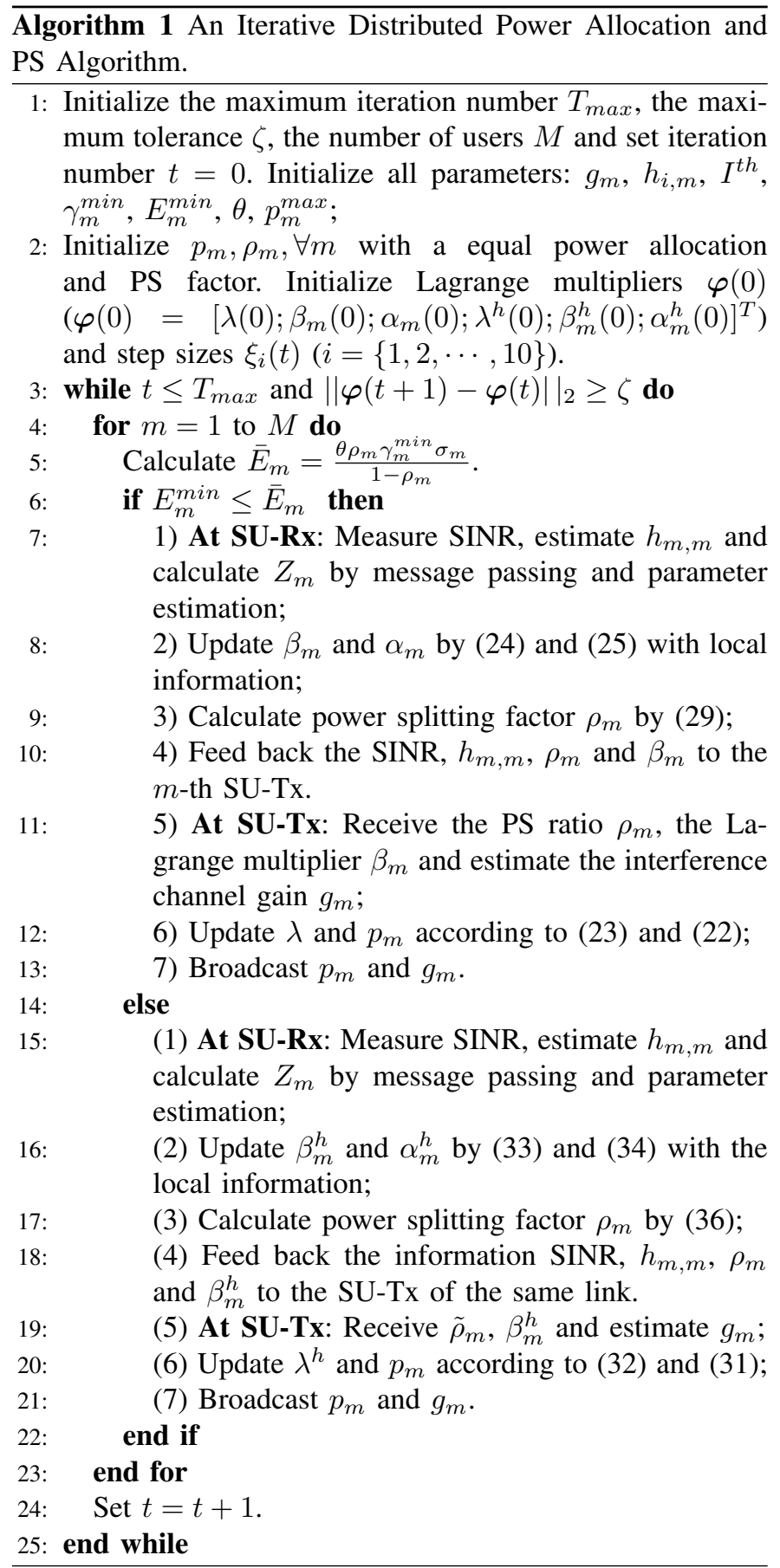

basic SINR of each SU will receive serious impact from the multiple access interference and effective signal of SUs. The reason is that the number of SUs often is much larger than that of PUs. As a result, the uncertainties of channel gains can be described as

$$
\left\{\begin{array}{l}
h_{i, m}=\bar{h}_{i, m}+\Delta h_{i, m}, \\
g_{m}=\bar{g}_{m}+\Delta g_{m} .
\end{array}\right.
$$

This is additive uncertainty error model [36]. $\Delta h_{i, m}$ and $\Delta g_{m}$ are the channel estimation errors which are uncertain terms. $\bar{h}_{i, m}$ and $\bar{g}_{m}$ are the estimated channel gains which usually are known values for SUs. Therefore, we only need to consider the uncertain terms and design the robust algorithm according to the uncertainty sets of $\Delta h_{i, m}$ and $\Delta g_{m}$.

Since the statistical distribution function of channel estimation errors are usually difficult to obtain [37], thus we model the uncertain parameters as the bounded channel uncertainty sets. Based on the spherical uncertainty formulation [38], channel uncertainties can be modeled by

$$
\begin{gathered}
\mathcal{R}_{g}=\{\boldsymbol{g} \mid\|\boldsymbol{g}-\overline{\boldsymbol{g}}\| \leq \tau\}, \\
\mathcal{R}_{h}=\left\{\boldsymbol{h}_{m} \mid\left\|\boldsymbol{h}_{m}-\overline{\boldsymbol{h}}_{m}\right\| \leq \omega_{m}\right\},
\end{gathered}
$$

where $\mathcal{R}_{g}$ and $\mathcal{R}_{h}$ denote the uncertainty sets. $\tau \geq$ 0 and $\omega_{m} \geq 0$ are the upper bounds of channel uncertainties of SU-to-PU links and SU-to-SU links, respectively. $\overline{\boldsymbol{g}}=\left[\bar{g}_{1}, \bar{g}_{2}, \cdots, \bar{g}_{M}\right]^{T}, \forall m$ and $\overline{\boldsymbol{h}}_{m}=$ $\left[\bar{h}_{1, m}, \bar{h}_{2, m}, \cdots, \bar{h}_{M, m}\right]^{T}, \forall i \neq m$.

Remark 3: Obviously, the uncertainty set (40) can be also reformulated as $\sum_{m=1}^{M} \Delta g_{m}^{2} \leq \tau^{2}$. Similarly, the constraint (41) is equivalent to $\sum_{i=1, i \neq m}^{M} \Delta h_{i, m}^{2} \leq \omega_{m}^{2}$. From the aspect of communication system design, the constraint (40) is used to limit the upper bound of the sum variance of channel uncertainties of SU-to-PU links $\left(\Delta g_{m}\right)$. This worst-case approach can bring a benefit, namely, the designed transmit power do not need to dynamically adjust according to the random channel uncertainty $\Delta g_{m}, \forall m$ only if all uncertainties do not extend the uncertainty set formulated in (40). This method is superior to the multiplicative uncertainty approach (e.g., $g_{m}=\bar{g}_{m}+\varepsilon \bar{g}_{m}$ ) [39], which requires to adjust the transmit power according to the time-varying uncertainty part. Under this case, it costs more energy and computational resource to update the optimal power.

\section{B. Transformation of Robust Constraint}

Based on the worst-case approach and the CauchyBuniakowsky-Schwarz inequality [38], the interference temperature constraint $C_{1}$ with channel uncertainties can be reformulated as

$$
\begin{gathered}
\max _{g_{m} \in \mathcal{R}_{g}} \sum_{m=1}^{M} p_{m} g_{m} \leq \sum_{m=1}^{M} p_{m} \bar{g}_{m}+\sqrt{\sum_{m=1}^{M} p_{m}^{2}} \sqrt{\sum_{m=1}^{M} \Delta g_{m}^{2}} \\
\leq \sum_{m=1}^{M} p_{m} \bar{g}_{m}+\sum_{m=1}^{M} p_{m} \tau \\
=\sum_{m=1}^{M} p_{m}\left(\bar{g}_{m}+\tau\right) \leq I^{t h} .
\end{gathered}
$$

Similarly, the worst-case SINR of each SU is given by

$$
\begin{aligned}
& \min _{\Delta h_{i, m} \in \mathcal{R}_{h}} \gamma_{m} \geq \gamma_{m}^{\min } \\
& \Leftrightarrow \frac{\left(1-\rho_{m}\right) \min _{\Delta h_{m}, m}\left(p_{m} h_{m, m}\right)}{\left(1-\rho_{m}\right) \max _{\Delta h_{i, m}}\left(\sum_{i \neq m}^{M} p_{i} h_{i, m}\right)+\sigma_{m}} \geq \gamma_{m}^{m i n} .
\end{aligned}
$$

Since the channel gain $h_{m, m}$ can be well estimated by the corresponding SU-Rx, therefore the estimation error is very small [41], where the estimated channel gain $\bar{h}_{m, m}$ can be fed back to the SU-Tx by the feeback channel. Based on the same approach used in (42). The SINR constraint with the 
consideration of uncertainty is given by

$$
\frac{\left(1-\rho_{m}\right) p_{m} \bar{h}_{m, m}}{\left(1-\rho_{m}\right) \sum_{i \neq m}^{M} p_{i}\left(\bar{h}_{i, m}+\omega_{m}\right)+\sigma_{m}} \geq \gamma_{m}^{\text {min }} .
$$

Based on the same approach in Section IV, the analytical solutions of robust RA algorithm under the lower-energy level are

$$
\begin{gathered}
p_{m}^{r, *}=\sqrt{\frac{\alpha_{m}^{r}}{\bar{h}_{m, m}\left(1+\beta_{m}^{r}+\lambda^{r}\left(\bar{g}_{m}+\tau\right)\right)}}, \\
\rho_{m}^{r, *}=\max \left(0, \sqrt{\hat{\beta}_{m}^{p s} / \hat{\lambda}_{m}^{p s}}\right),
\end{gathered}
$$

where the Lagrange multipliers $\alpha_{m}^{r}, \beta_{m}^{r}, \lambda^{r}, \hat{\beta}_{m}^{p s}$ and $\hat{\lambda}_{m}^{p s}$ can be updated by the same approach.

Remark 4: Since the PS subproblem under the low-energy level is without relationship with uncertainties and transmit power so that the value is the same as the non-robust case, i.e., $\rho_{m}^{r, *}=\rho_{m}^{*}$. And the robust power allocation under this case is only affected by the channel uncertainties of the SUto-PU links. Therefore, it can overcome any uncertainties of SU's links. Moreover, based on the same method, we can get the robust RA solutions for the high-energy level. Meanwhile, the optimal solutions are not affected by the minimum EH threshold.

\section{Robust Sensitivity Analysis}

In order to determine the impact of uncertain parameters on the whole system performance, the performance gap of the non-robust RA problem (3) and the robust RA problem with the constraints of (42) and (44) is analyzed under the cases of low-energy level and high-energy level.

1) Case 1-low energy level: : From the subproblem (16), it is obvious that only two parameters may be uncertain, such as the channel gain between SUs and PUs (e.g., $g_{m}$ ) and the direct channel gain between the SU-Tx and the corresponding SU-Rx (e.g., $h_{m, m}$ ). Combining the model (16) with (42), we can construct the following function

$$
F^{\text {robust }} \triangleq \min _{p_{m}}\left\{\sum_{m=1}^{M} p_{m}+\lambda^{r}\left(\sum_{m=1}^{M} p_{m}\left(\bar{g}_{m}+\tau\right)-I^{t h}\right)\right.
$$

$\left.+\sum_{m=1}^{M} \beta_{m}^{r}\left(p_{m}-p_{m}^{\max }\right)+\sum_{m=1}^{M} \alpha_{m}^{r}\left(\frac{1}{p_{m} \bar{h}_{m, m}}-\frac{1-\rho_{m}}{\gamma_{m}^{\min } \sigma_{m}}\right)\right\}$

When the uncertain parameter is very small, the optimal power allocation and Lagrange multipliers can be assumed to be the same values (e.g., $\lambda^{r} \approx \lambda$ ). Assume the optimal power and Lagrange multiplier are $p_{m}^{r, *}$ and $\lambda^{r, *}$. Since the non-robust RA algorithm assumes the estimated channel gain is equal to the true value, namely, $\bar{g}_{m}=g_{m}$, based on the sensitivity principle [40], (47) can be approximated as

$$
F^{\text {robust }} \triangleq F^{\text {non-robust }}+\lambda^{r, *} \sum_{m=1}^{M} p_{m}^{r, *} \tau .
$$

The performance gap is

$$
G_{\text {low }}=\lambda^{r, *} \sum_{m=1}^{M} p_{m}^{r, *} \tau .
$$

Since the parameters in (49) are non-negative, we can obtain an important result, namely, the total power consumption under the robust RA algorithm is bigger than that of the nonrobust algorithm. In other words, the robust RA algorithm can overcome the outage probability in cost of more power consumption. From (26), it is clear that the PS coefficient cannot be directly influenced by the uncertainties due to no uncertainty in the system model (26). Moreover, when the upper bound of channel estimation error becomes bigger, the gap becomes larger.

2) Case 2-high energy level: : From the subproblem (30), based on the same idea, we can construct the following function

$$
\begin{aligned}
& J^{\text {robust }} \triangleq \min _{p_{m}}\left\{\sum_{m=1}^{M} p_{m}+\lambda^{r}\left(\sum_{m=1}^{M} p_{m}\left(\bar{g}_{m}+\tau\right)-I^{\text {th }}\right)\right. \\
& +\sum_{m=1}^{M} \beta_{m}^{r}\left(p_{m}-p_{m}^{\max }\right)+\sum_{m=1}^{M} \alpha_{m}^{r}\left(\frac{\gamma_{m}^{m i n} \sigma_{m}-\left(1-\rho_{m}\right) Z_{m}}{p_{m} h_{m, m}}\right. \\
& \left.\left.-\left(1-\rho_{m}\right)\right)\right\} .
\end{aligned}
$$

Define the optimal solutions as $p_{m}^{r, *}, \rho_{m}^{r, *}$ and the optimal Lagrange multipliers as $\lambda^{r, *}$ and $\alpha_{m}^{r, *}$. (50) can be rewritten as

$$
\begin{aligned}
& J^{\text {robust }} \triangleq\left\{\min _{p_{m}} \sum_{m=1}^{M} p_{m}+\lambda^{r}\left(\sum_{m=1}^{M} p_{m} \bar{g}_{m}-I^{\text {th }}\right)\right. \\
& +\sum_{m=1}^{M} \beta_{m}^{r}\left(p_{m}-p_{m}^{\max }\right)-\sum_{m=1}^{M} \alpha_{m}^{r}\left(1-\rho_{m}\right) \\
& \left.+\sum_{m=1}^{M} \frac{\alpha_{m}^{r} \gamma_{m}^{m i n} \sigma_{m}}{p_{m} h_{m, m}}-\sum_{m=1}^{M} \frac{\alpha_{m}^{r}\left(1-\rho_{m}\right) \sum_{i \neq m}^{M} p_{i} \bar{h}_{i, m}}{p_{m} \bar{h}_{m, m}}\right\} \\
& +\lambda^{r} \sum_{m=1}^{M} p_{m} \tau-\sum_{m=1}^{M} \frac{\alpha_{m}^{r}\left(1-\rho_{m}\right)\left(\sum_{i \neq m}^{M} p_{i} \omega_{m}\right)}{p_{m} \bar{h}_{m, m}} .
\end{aligned}
$$

And the performance gap is

$$
G_{h i g h}=\lambda^{r, *} \sum_{m=1}^{M} p_{m}^{r, *} \tau-\left(\sum_{i \neq m}^{M} p_{i}^{r, *}\right) \sum_{m=1}^{M} \frac{\alpha_{m}^{r, *} \omega_{m}\left(1-\rho_{m}^{r, *}\right)}{p_{m}^{r * *} \bar{h}_{m, m}} .
$$

From (52), it is difficult to directly determine whether the gap $G_{\text {high }}$ is greater than zero or not. Based on (44), we have the following relationship

$$
\frac{\left(1-\rho_{m}^{r, *}\right) \sum_{i \neq m}^{M} p_{i}^{r, *}}{p_{m}^{r, *} \bar{h}_{m, m}}+\frac{\sigma_{m}}{p_{m}^{r * *} \bar{h}_{m, m}} \leq \frac{1-\rho_{m}^{r, *}}{\gamma_{m}^{m i n}}
$$

Since the background noise $\sigma_{m}$ is much smaller than the mutual interference of users (e.g., $\sum_{i \neq m}^{M} p_{i}^{r, *}$ ) in practical systems, the second item of (53) can be ignored. Under the high energy level, the optimal PS factor must satisfy $\rho_{m}^{r * *}>0.5$ according to the feasible region analysis in Section III. Thus, combining 
(52) with (53), we have

$$
\begin{aligned}
G_{h i g h} & \geq \sum_{m=1}^{M}\left\{\lambda^{r, *} p_{m}^{r, *} \tau-\alpha_{m}^{r, *} \frac{\omega_{m}\left(1-\rho_{m}^{r, *}\right)}{\gamma_{m}^{\min }}\right\} \\
\geq & \sum_{m=1}^{M}\left\{\lambda^{r, *} p_{m}^{r, *} \tau-\alpha_{m}^{r, *} \frac{\omega_{m}}{2 \gamma_{m}^{m i n}}\right\} .
\end{aligned}
$$

When the relationship $p_{m}^{r, *} \geq \frac{\alpha_{m}^{r * *} \omega_{m}}{2 \gamma_{m i n}^{m i n} \lambda^{r, *} \tau}$ holds, $G_{h i g h} \geq 0$, otherwise $G_{\text {high }}<0$. Additionally, since the upper bound of the channel estimation error of link $m$ is very small by comparing with $2 \gamma_{m}^{\min }$, (54) can be simplified as $G_{h i g h} \geq$ $\sum_{m=1}^{M} \lambda^{r, *} p_{m}^{r, *} \tau \geq 0$. If we do not know the parameter information in (54), the value of $G_{h i g h}$ can be determined by the orthogonality of constraint [34].

According to the orthogonality of the optimal value and the constraint [34], we have

$$
\begin{gathered}
\lambda^{r, *} \times\left(\sum_{m=1}^{M} p_{m}^{r, *}\left(\bar{g}_{m}+\tau\right)-I^{t h}\right)=0 \\
\alpha_{m}^{r, *} \times\left(\frac{\theta \rho_{m}^{r, *} \gamma_{m}^{m i n} \sigma_{m}-\left(1-\rho_{m}\right) Z_{m}}{p_{m}^{r, *} \bar{h}_{m, m}}-\frac{1}{1-\rho_{m}^{r, *}}\right)=0
\end{gathered}
$$

Case 1: when the robust interference constraint $\sum_{m=1}^{M} p_{m}^{r, *}\left(\bar{g}_{m}+\tau\right)<I^{t h}$ holds and the robust SINR constraint takes the equal sign, the optimal Lagrange multipliers are $\lambda^{r, *}=0$ and $\alpha_{m}^{r, *}>0$. Thus $G_{h i g h} \leq 0$ holds, the total power consumption under the robust RA scheme is smaller than that of the non-robust scheme.

Case 2: when the robust interference constraint $\sum_{m=1}^{M} p_{m}^{r, *}\left(\bar{g}_{m}+\tau\right)=I^{t h}$ and the robust SINR constraint holds (e.g., $\gamma_{m}\left(p_{m}^{r, *}, \omega_{m}\right)>\gamma_{m}^{\min }$ ), the optimal Lagrange multipliers are $\lambda^{r, *}>0$ and $\alpha_{m}^{r, *}=0$. Thus $G_{h i g h} \geq 0$ holds, the total power consumption under the robust RA scheme is bigger than that of the non-robust scheme.

Case 3: when both the robust interference constraint (55) and the robust SINR constraint (56) take the equal sign, the optimal Lagrange multipliers are $\lambda^{r, *}>0$ and $\alpha_{m}^{r, *}>0$. The value of $G_{h i g h}$ can not be determined by this method and the above approach can be used in this case.

Case 4: when the robust interference constraint $\sum_{m=1}^{M} p_{m}^{r, *}\left(\bar{g}_{m}+\tau\right)<I^{t h}$ holds and the robust SINR constraint holds (e.g., $\gamma_{m}\left(p_{m}^{r, *}, \omega_{m}\right)>\gamma_{m}^{\min }$ ), the optimal Lagrange multipliers are $\lambda^{r, *}=0$ and $\alpha_{m}^{r * *}=0$. The total power consumption of the robust RA algorithm and the non-robust algorithm is the same since the optimal power is $p_{m}^{*}=p_{m}^{\max }$ which is a channel independent parameter.

\section{Simulation Results}

In this section, the performance of the proposed RA schemes is evaluated by the simulation results. To verify the effectiveness of our algorithm, the following results are provided by comparing with the existing algorithms. All simulation results are executed by using Matlab 2016a. The $\mathrm{CPU}$ is Intel Core $\mathrm{i7}-6500 \mathrm{U}$, the frequency is $2.59 \mathrm{GHz}$. The optimal and robust RA algorithm are defined as 'Our
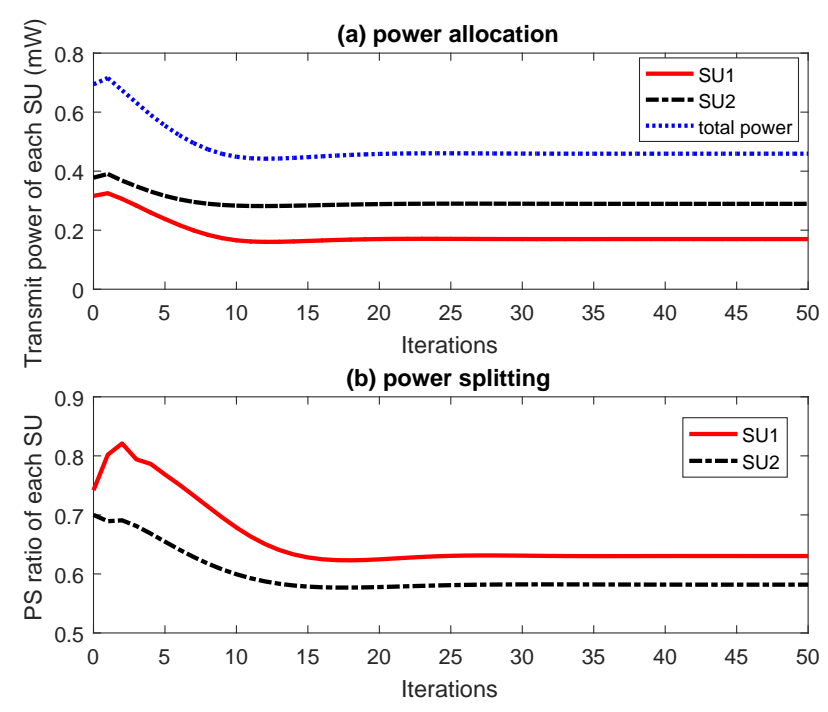

Fig. 1. The Converge of the proposed algorithm.

non-robust scheme with SWIPT' and 'Our robust scheme with SWIPT'. The robust algorithm without SWIPT in [41] is defined as 'Robust scheme without SWIPT'. The optimal RA algorithm with SWIPT is defined as 'Non-robust without SWIPT' [42]. The maximum transmit power of each SU is $p_{m}^{\max }=1 \mathrm{~mW}$. The background noise is $\sigma_{m}=10^{-8} \mathrm{~mW}$. The interference power threshold of PU is $I^{t h}=1 \times 10^{-6} \mathrm{~mW}$. The values of estimated channel gains are randomly generated from the interval $(0,1)$ and the channel uncertainty is bounded by $[0,0.2]$ [33]. The energy conversion efficiency is $\theta=1$ according to [24].

\section{A. Performance Evaluation}

Fig. 1 shows the coverage performance of our algorithm. Assume there are two SUs $(M=2)$, and the channel gain of SU1 is better than that of SU2. The SINR threshold is $\gamma_{m}^{\min }=2 \mathrm{~dB}$. According to Fig. 1, we can easily find that the transmission power and PS ratios of all SUs can quickly achieve the optimal values under 20 iterations. The power consumption of SU1 is obviously lower than that of SU2. The reason is that under the same QoS requirement, the user with good channel gain can consume less power to meet the performance need and save energy. Furthermore, the PS ratio of SU2 is higher than that of SU1. Since the higher PS means more information required at the receiver so that SU2 requires more transmit power to ensure the basic SINR.

Fig. 2 presents the sum transmit power of SUs versus the minimum harvested energy threshold under different SINR threshold. It shows the total power consumption becomes bigger under the increasing $E_{m}^{m i n}$ for different SINR requirements $\gamma_{m}^{\min }$. Additionally, the higher SINR threshold is, the bigger sum power consumption will be. Since SUs try their best to improve the power to ensure the normal communication of all links. With the increasing SINR requirement, each SU will adjust its power to satisfy the basic SINR.

Fig. 3 gives the power splitting versus the minimum harvested energy threshold under different SINR thresholds. It is 


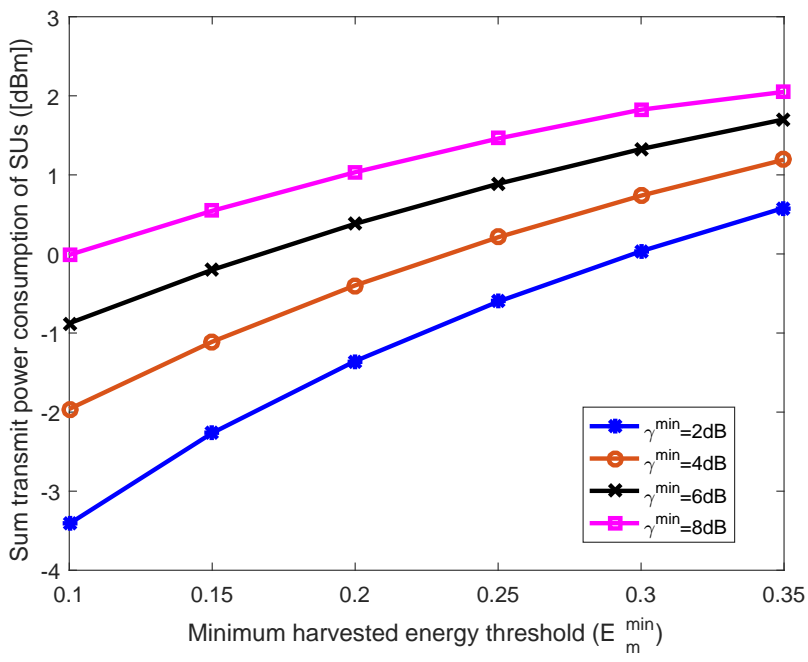

Fig. 2. Sum power consumption versus minimum required harvested energy.

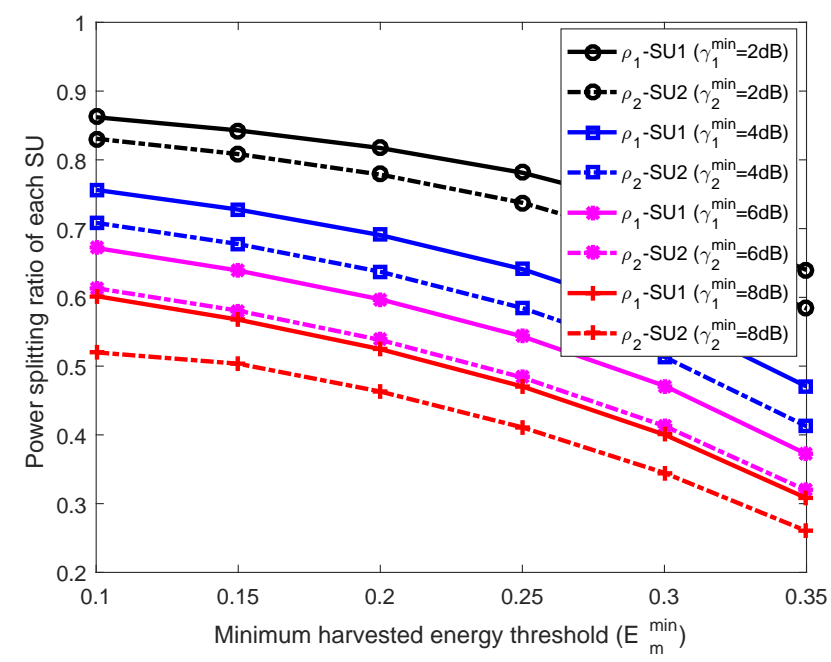

Fig. 3. The PS versus the minimum harvested energy threshold.

clear that the power splitting ratio of each decreases with the increasing minimum harvested energy requirement $E_{m}^{\min }$ and the increasing SINR $\gamma_{m}^{\min }$ because the increasing harvested energy means storing more energy so that the minimum required transmit power becomes bigger. Furthermore, in order to achieve total power minimization, the decreasing power splitting ratios can further reduce power consumption.

Fig. 4 shows the total power consumption versus the QoS threshold of SU under different interference temperature levels. The minimum harvested energy is $E_{m}^{\min }=0.2$. From the figure, we know that the sum transmit power of SUs increases with the increasing SINR threshold. Moreover, the total transmission power of the secondary system under the bigger $I^{\text {th }}$ is higher than that under the small one. Because the bigger interference temperature level extends the upper bound of transmit power so that it can improve the transmit power of SUs and rebuild the communication link of SUs with bad channel environments. Additionally, it also increases the opportunity of user's access.

Fig. 5 presents the sum transmit power of SUs versus the

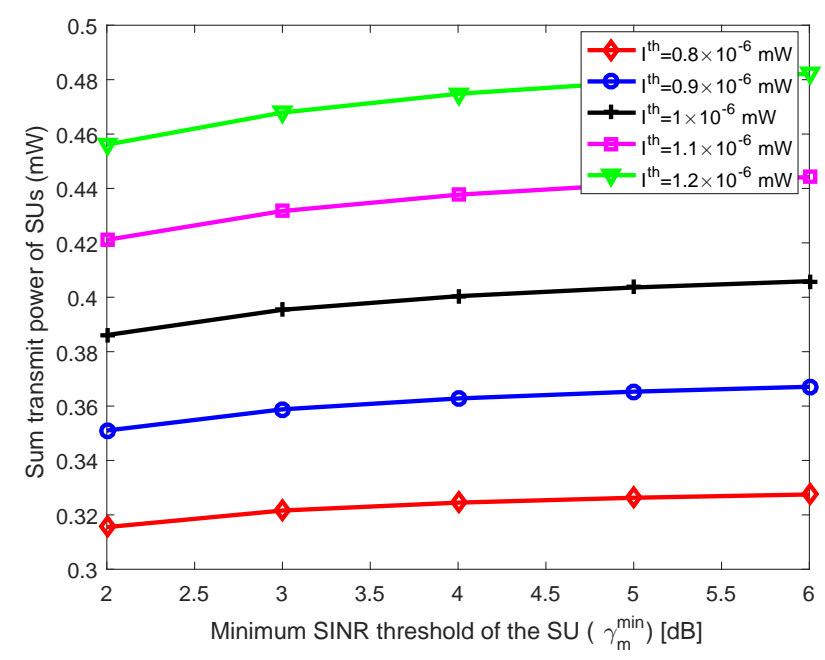

Fig. 4. Sum transmit power of SUs versus the minimum SINR threshold.

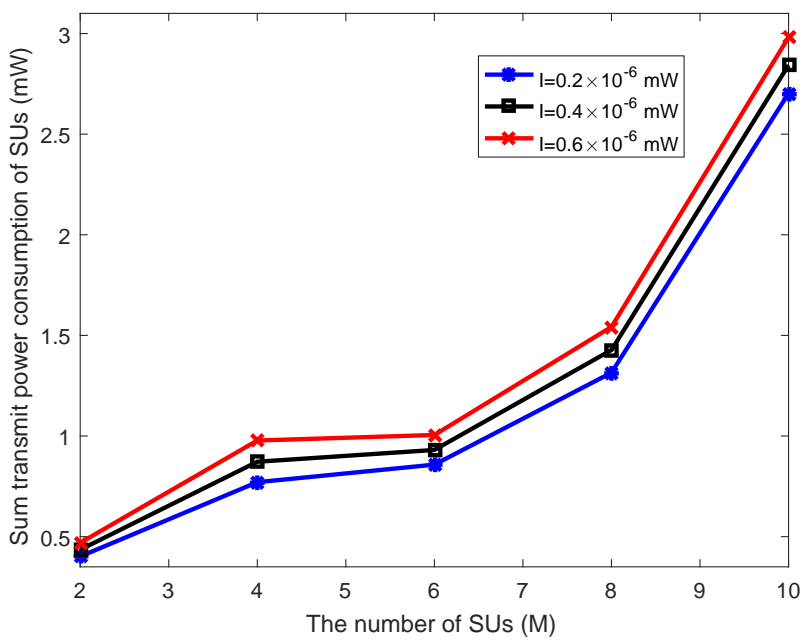

Fig. 5. Sum transmit power of SUs versus the number of SUs.

number of SUs under different PU's interference. Apparently, the total transmission power of SUs becomes bigger with the increasing number of SUs. Since more SUs access the network and share spectrum resource with others, therefore, the total power consumption of the network becomes bigger. Additionally, the gap of total transmit power under the big number of SUs is much bigger than that under the small number of SUs. Because more SUs will increase more mutual interference among SUs so that each SU tries to improve its transmit power for overcoming the effect of harmful interference. Moreover, the interference from PU will become very large due to the introduced interference over each SU's link.

\section{B. Algorithm Comparison}

According to the definition of EE (e.g., bits/J) in [43] (i.e, sum data rate over sum power consumption), we define the following $\mathrm{EE}, \eta^{\text {swipt }}=$ $\sum_{m=1}^{M} \log _{2}\left(1+\gamma_{m}\left(p_{m}, \rho_{m}\right)\right) /\left(\sum_{m=1}^{M} p_{m}+P_{c}-\sum_{m=1}^{M} E_{m}\right) \quad$ and 


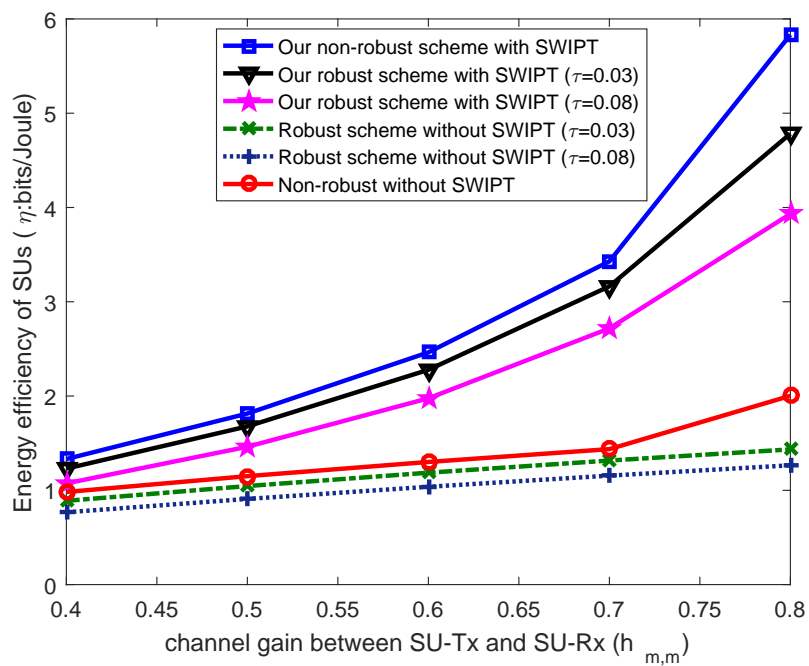

Fig. 6. Total EE of SUs versus the channel gain of SU's link.

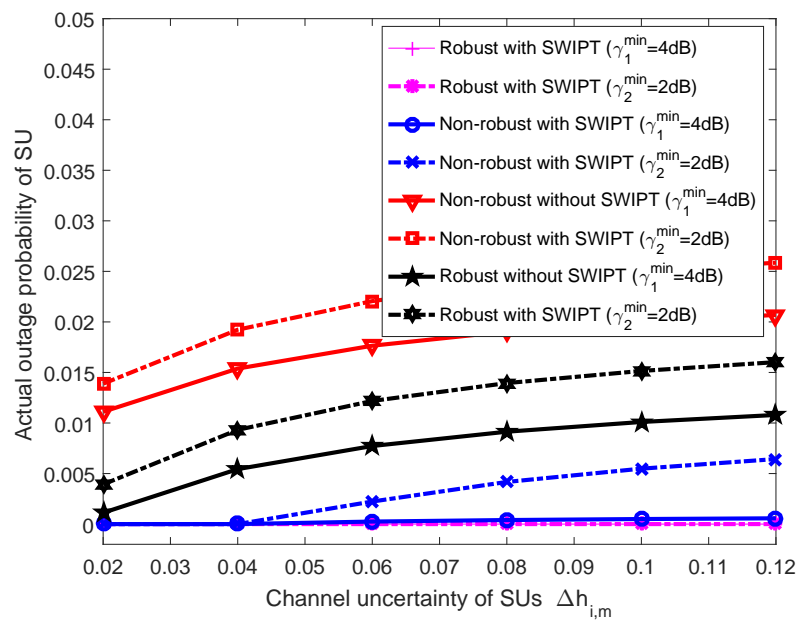

Fig. 7. Total EE of SUs versus the channel gain of SU's link.

$\eta_{\text {without }}^{\text {swipt }}=\sum_{m=1}^{M} \log _{2}\left(1+\gamma_{m}\left(p_{m}\right)\right) /\left(\sum_{m=1}^{M} p_{m}+P_{c}\right)$. And $P_{c}$ is a constant which denotes the circuit power consumption.

Fig. 6 shows the EE of SUs versus the direct channel gain. It is observed that the total EE becomes bigger with the increasing channel gain between SU-Tx and SU-Rx. This is due to the fact that a bigger channel gain results in more transmit power to keep the basic SINR requirement. Besides, Our schemes with SWIPT have a good performance than other algorithms. The reason is that the harvested energy can compensate for the energy requirement of the system. Moreover, with the bigger $\tau$ (e.g., the upper bound of uncertainty), the total EE decreases to give better protection to the PU.

In Fig. 7, we study the robustness of (e.g., user's outage probability) algorithms under channel uncertainties. The outage probability of $\mathrm{SU}$ can be defined as $\operatorname{Pr}($ out $)=$ $\max \left\{0,\left(\gamma_{m}^{\min }-\gamma_{m}\right) / \gamma_{m}^{\min }\right\}$. From the figure, it is clear that the actual outage probability of SU improves a lot with the big uncertainty. But our proposed algorithms can overcome the effect of uncertainty and have a small outage probability. The non-robust algorithm without SWIPT has the biggest outage probability. The reason is that the type of algorithm does not consider the uncertainty in the design of the RA algorithm ahead of time. With the increasing of uncertainty $\Delta h_{m, m}$, the outage probability of SUs cannot increase indefinitely since the available transmission power of SU is constrained by both the maximum transmission power and the interference temperature level.

\section{CONCLUSIONS}

In this paper, we have proposed a RA strategy to solve the total power minimization problem in SWIPT-enabled cognitive networks under perfect CSI and imperfect CSI, respectively, in a distributed way. In particular, we have formulated the joint power allocation and PS problems for the secondary links as the optimization problems with the QoS constraints of SUs and the interference power constraint of PU. Also, imperfect CSI is considered in the analysis and robust algorithm design. The optimal solutions are obtained under the low-harvested energy requirement and the high-harvested energy requirement, respectively. Based on the bounded channel uncertainties and the worst-case principle, the robust RA problem is also analyzed. Simulation results demonstrated that the proposed algorithm has good convergence performance, EE, and the robustness by comparing with the existing algorithms. Our RA strategy can be used in low-power networks (e.g., IoT, wireless sensor networks or D2D networks) for prolonging the lifetime and saving energy consumption. Moreover, our work can also be extended to the scenarios of EE maximization and the nonlinear EH model.

\section{APPENDIX A \\ PROOF OF (4)}

Define $Z_{m}=\sum_{i \neq m}^{M} p_{i} h_{i, m}$, the constraint $C_{2}$ can be rewritten as

$$
p_{m} h_{m, m} \geq \gamma_{m}^{\min } Z_{m}+\gamma_{m}^{\min } \frac{\sigma_{m}}{1-\rho_{m}} .
$$

The Eq. (57) can be reformulated as

$$
\frac{p_{m} h_{m, m}}{\gamma_{m}^{\min }}-\frac{\sigma_{m}}{1-\rho_{m}} \geq Z_{m}
$$

Since $Z_{m} \geq 0$, we have

$$
p_{m} h_{m, m} \geq \frac{\gamma_{m}^{m i n} \sigma_{m}}{1-\rho_{m}} .
$$

At the same time, the constraint $C_{3}$ can be rewritten as

$$
p_{m} h_{m, m} \geq \frac{E_{m}^{\text {min }}}{\theta \rho_{m}}-Z_{m} .
$$

Based on $(57)+\gamma_{m}^{\min } \times(60)$, we have

$$
p_{m} h_{m, m} \geq H_{m},
$$

where $H_{m}=\frac{\gamma_{m}^{\min }}{1+\gamma_{m}^{\min }}\left(\frac{\sigma_{m}}{1-\rho_{m}}+\frac{E_{m}^{\min }}{\theta \rho_{m}}\right)$.

According to (59) and (61), we have

$$
p_{m} h_{m, m} \geq \bar{H}_{m},
$$


where $\bar{H}_{m}=\max \left(H_{m}, \frac{\gamma_{m}^{\min } \sigma_{m}}{1-\rho_{m}}\right)$. Therefore,

$$
\begin{aligned}
H_{m}-\frac{\gamma_{m}^{\min } \sigma_{m}}{1-\rho_{m}} & =\frac{\gamma_{m}^{\min }}{1+\gamma_{m}^{\min }}\left(\frac{\sigma_{m}}{1-\rho_{m}}+\frac{E_{m}^{\min }}{\theta \rho_{m}}\right)-\frac{\gamma_{m}^{\min } \sigma_{m}}{1-\rho_{m}} \\
& =\frac{\gamma_{m}^{\min }}{1+\gamma_{m}^{\min }}\left(\frac{E_{m}^{\min }\left(1-\rho_{m}\right)-\theta \rho_{m} \gamma_{m}^{m i n} \sigma_{m}}{\theta \rho_{m}\left(1-\rho_{m}\right)}\right) \\
& \left\{\begin{array}{l}
\leq 0, E_{m}^{\min } \leq\left(\theta \rho_{m} \gamma_{m}^{\min } \sigma_{m}\right) /\left(1-\rho_{m}\right), \\
0, \text { otherwise. }
\end{array}\right.
\end{aligned}
$$

According to (62) and (63), we have

$$
p_{m} h_{m, m} \geq \bar{H}_{m}=\left\{\begin{array}{l}
\frac{\gamma_{m}^{m i n} \sigma_{m}}{1-\rho_{m}}, E_{m}^{m i n} \leq \frac{\theta \rho_{m} \gamma_{m}^{m i n} \sigma_{m}}{1-\rho_{m}}, \\
H_{m}, \text { otherwise. }
\end{array}\right.
$$

The proof is completed.

\section{APPENDIX B \\ PROOF OF (14)}

We use the counter-evidence approach to proof the equation. As a result, we have

$$
\begin{aligned}
& \frac{E_{m}^{\text {min }}}{\theta \rho_{m}}-Z_{m} \geq H_{m} \\
& \Leftrightarrow \frac{1}{1+\gamma_{m}^{\min }} \frac{E_{m}^{\text {min }}}{\theta \rho_{m}} \geq Z_{m}+\frac{\gamma_{m}^{\min }}{1+\gamma_{m}^{\min }} \frac{\sigma_{m}}{1-\rho_{m}} \\
& \Leftrightarrow \frac{E_{m}^{\text {min }}}{\theta \rho_{m}} \geq\left(1+\gamma_{m}^{\text {min }}\right) Z_{m}+\gamma_{m}^{\min } \frac{\sigma_{m}}{1-\rho_{m}} .
\end{aligned}
$$

According to $C_{3}$, we have

$$
Z_{m} \geq \frac{E_{m}^{m i n}}{\theta \rho_{m}}-p_{m} h_{m, m}
$$

Combining (65) with (66), we have

$$
\frac{E_{m}^{m i n}}{\theta \rho_{m}} \geq\left(1+\gamma_{m}^{m i n}\right)\left(\frac{E_{m}^{m i n}}{\theta \rho_{m}}-p_{m} h_{m, m}\right)+\gamma_{m}^{\text {min }} \frac{\sigma_{m}}{1-\rho_{m}} .
$$

Based on (67), we have

$$
\left(1+\gamma_{m}^{m i n}\right) p_{m} h_{m, m} \geq \gamma_{m}^{m i n} \frac{E_{m}^{m i n}}{\theta \rho_{m}}+\gamma_{m}^{m i n} \frac{\sigma_{m}}{1-\rho_{m}} .
$$

Since $\frac{E_{m}^{\min }}{\theta \rho_{m}} \leq Z_{m}+p_{m} h_{m, m}$ holds, we assume that the low bound of left side is bigger than the upper bound of right side in (68) under the worst case. Thus we have

$$
\left(1+\gamma_{m}^{m i n}\right) p_{m} h_{m, m} \geq \gamma_{m}^{\min }\left(Z_{m}+p_{m} h_{m, m}\right)+\gamma_{m}^{\min } \frac{\sigma_{m}}{1-\rho_{m}} .
$$

According to $C_{3}$, we have

$$
\begin{aligned}
& p_{m} h_{m, m} \geq \gamma_{m}^{\text {min }} Z_{m}+\gamma_{m}^{\text {min }} \frac{\sigma_{m}}{1-\rho_{m}} \\
& \Leftrightarrow \frac{p_{m} h_{m, m}}{Z_{m}+\frac{\sigma_{m}}{1-\rho_{m}}} \geq \gamma_{m}^{\text {min }} \\
& \Leftrightarrow \frac{\left(1-\rho_{m}\right) p_{m} h_{m, m}}{\left(1-\rho_{m}\right) Z_{m}+\sigma_{m}} \geq \gamma_{m}^{\text {min }} \\
& \Leftrightarrow \frac{\left(1-\rho_{m}\right) p_{m} h_{m, m}}{\left(1-\rho_{m}\right)\left(\sum_{i \neq m}^{M} p_{i} h_{i, m}\right)+\sigma_{m}} \geq \gamma_{m}^{\min } .
\end{aligned}
$$

According to the definition of SINR, we have

$$
\gamma_{m} \geq \gamma_{m}^{\min }
$$

Obviously, the above equation holds for each user $\forall m$. Therefore,

$$
\frac{E_{m}^{\min }}{\theta \rho_{m}}-Z_{m} \geq H_{m}
$$

The proof is completed.

\section{REFERENCES}

[1] Z. Na, J. Lv, F. Jiang, M. Xiong, and N. Zhao, "Joint subcarrier and subsymbol allocation-based simultaneous wireless information and power transfer for multiuser GFDM in IoT," IEEE Internet Things J., vol. 6, no. 4, pp. 5999-6006, Aug. 2019.

[2] T. Perera, D. Jayakody, S. Sharma, S. Chatzinotas, and J. Li, "Simultaneous wireless information and power transfer (SWIPT): Recent advances and future challenges," IEEE Commun. Sur. \& Tut., vol. 20, no. 1, pp. 264-302, Jan. 2018.

[3] Y. Xu, G. Li, Y. Yang, M. Liu and G. Gui, "Robust resource allocation and power splitting in SWIPT enabled heterogeneous networks: a robust minimax approach," IEEE Internet of Things J., vol. 6, no. 6, pp. 1079910811, Dec. 2019

[4] W. Guo, S. Zhou, Y. Chen, S. Wang, X. Chu, and Z. Niu, "Simultaneous information and energy flow for IoT relay systems with crowd harvesting," IEEE Commun. Mag., vol. 54, no. 11, pp. 143-149, Nov. 2016.

[5] H. Zhang, J. Du, J. Cheng, K. Long and V. C. M. Leung, "Incomplete CSI based resource optimization in SWIPT enabled heterogeneous networks: a non-cooperative game theoretic approach," IEEE Trans. Wireless Commun., vol. 17, no. 3, pp. 1882-1892, Mar. 2018.

[6] W. Wu, X. Yin, P. Deng, T. Guo and B. Wang, "Transceiver design for downlink SWIPT NOMA systems with cooperative full-duplex relaying," IEEE Access, vol. 7, pp. 33464-33472, Mar. 2019.

[7] G. Yang, W. P. Tay, Y. L. Guan and Y.-C. Liang, "Optimal power allocation for diffusion-type sensor networks with wireless information and power transfer," IEEE Access, vol. 7, pp. 32408-32422, Mar. 2019.

[8] Y. Xu, G. Li, Q. Liu and Z. Wang, "Resource allocation for OFDMA-based cognitive networks: an interference-efficient perspective," VTC2019-Fall, Honolulu, 2019, pp. 1-5.

[9] M. Liu, J. Yang, T. Song, J. Hu, and G. Gui, "Deep learning-inspired message passing algorithm for efficient resource allocation in cognitive radio networks," IEEE Trans. Veh. Technol., vol. 69, no. 1, pp. 641-653, Jan. 2019.

[10] Y. Wang, M. Liu, J. Yang, G. Gui, "Data-driven deep learning for automatic modulation recognition in cognitive radios," IEEE Trans. Veh. Technol., vol. 68, no. 4, pp. 4074-4077, Apr. 2019.

[11] G. Gui, H. Sari, and E. Biglieri, "A new definition of fairness for nonorthogonal multiple access," IEEE Commun. Lett., vol. 23, no. 7, pp. 1267-1271, May 2019.

[12] M. Liu, J. Yang, and G. Gui, "DSF-NOMA: UAV-assisted emergency communication technology in a heterogeneous internet of things," IEEE Internet of Things J., vol. 6, no. 3, pp. 5508-5519, June 2019.

[13] S. Lee, R. Zhang and K. Huang, "Opportunistic wireless energy harvesting in cognitive radio networks," IEEE Trans. Wireless Commun., vol. 12, no. 9, pp. 4788-4799, Sept. 2013.

[14] T. Zhang, W. Chen and F. Yang, "Balancing delay and energy efficiency in energy harvesting cognitive radio networks: A stochastic stackelberg game approach," IEEE Trans. Cognitive Commun. Netw., vol. 3, no. 2, pp. 201-216, Jun. 2017

[15] D. Xu and Q. Li, "Joint power control and time allocation for wireless powered underlay cognitive radio networks," IEEE Wireless Commun. Lett., vol. 6, no. 3, pp. 294-297, Jun. 2017.

[16] Z. Hu, N. Wei and Z. Zhang, "Optimal resource allocation for harvested energy maximization in wideband cognitive radio network with SWIPT," IEEE Access, vol. 5, no. 1, pp. 23383-23394, Aug. 2017.

[17] X. Liu, F. Li and Z. Na, "Optimal resource allocation in simultaneous cooperative spectrum sensing and energy harvesting for multichannel cognitive radio," IEEE Access, vol. 5, no. 1, pp. 3801-3812, Mar. 2017.

[18] A. P. Shrestha and S. Yoo, "Optimal resource allocation using support vector machine for wireless power transfer in cognitive radio networks," IEEE Trans. Veh. Technol., vol. 67, no. 9, pp. 8525-8535, Sept. 2018. 
[19] Y. Cheng, P. Fu, Y. Ding, B. Li and X. Yuan, "Proportional fairness in cognitive wireless powered communication networks," IEEE Commun Lett., vol. 21, no. 6, pp. 1397-1400, Jun. 2017.

[20] Y. Wang, Y. Wang, F. Zhou, Y. Wu and H. Zhou, "Resource allocation in wireless powered cognitive radio networks based on a practical nonlinear energy harvesting model," IEEE Access, vol. 5, pp. 17618-17626, Jun. 2017

[21] D. $\mathrm{Xu}$ and Q. Li, "Resource allocation for secure communications in cooperative cognitive wireless powered communication networks," IEEE Syst. J., vol. 13, no. 3, pp. 2431-2442, Sept. 2019.

[22] C. Xu, Q. Zhang, Q. Li, Y. Tan and J. Qin, "Robust transceiver design for wireless information and power transmission in underlay MIMO cognitive radio networks," IEEE Commun. Lett., vol. 18, no. 9, pp. 1665 1668, Sept. 2014.

[23] D. W. K. Ng, E. S. Lo and R. Schober, "Multiobjective resource allocation for secure communication in cognitive radio networks with wireless information and power transfer," IEEE Trans. Veh. Technol. vol. 65, no. 5, pp. 3166-3184, May 2016.

[24] F. Zhou, Z. Li, J. Cheng, Q. Li and J. Si, "Robust AN-aided beamforming and power splitting design for secure MISO cognitive radio with SWIPT," IEEE Trans. Wireless Commun., vol. 16, no. 4, pp. 2450-2464, April 2017.

[25] F. Zhou, N. C. Beaulieu, J. Cheng, Z. Chu and Y. Wang, "Robust maxmin fairness resource allocation in sensing-based wideband cognitive radio with SWIPT: imperfect channel sensing," IEEE Syst. J., vol. 12, no. 3, pp. 2361-2372, Sept. 2018

[26] X. Zhang, Y. Wang, F. Zhou, N. Al-Dhahir and X. Deng, "Robust resource allocation for MISO cognitive radio networks under two practical non-linear energy harvesting models," IEEE Commun. Lett. vol. 22, no. 9, pp. 1874-1877, Sept. 2018

[27] R. Zhang and C. K. Ho, "MIMO broadcasting for simultaneous wireless information and power transfer," IEEE Trans. Wireless Commun., vol. 12, no. 5, pp. 1989-2001, May 2013.

[28] Y.-C. Liang, K. Chen, G. Y. Li and P. Mahonen, "Cognitive radio networking and communications: An overview," IEEE Trans. Veh. Technol., vol. 60, no. 7, pp. 3386-3407, Sept. 2011.

[29] Y. Xu and G. Li, "Optimal and robust interference efficiency maximization for multicell heterogeneous networks," IEEE Access, vol. 7, pp 102406-102416, Jul. 2019.

[30] H. Yu, S. Guo, Y. Yang and B. Xiao, "Optimal target secrecy rate and power allocation policy for a SWIPT system over a fading wiretap channel," IEEE Syst. J., vol. 12, no. 4, pp. 3291-3302, Dec. 2018.

[31] Z. Zhou, C. Gao, C. Xu, T. Chen, D. Zhang and S. Mumtaz, "Energyefficient stable matching for resource allocation in energy harvestingbased device-to-device communications," IEEE Access, vol. 5, pp 15184-15196, Mar. 2017.

[32] Y. Xu, X. Zhao and Y. Liang, "Robust Power control and beamforming in cognitive radio networks: a survey," IEEE Commun. Sur. \& Tut., vol. 17, no. 4, pp. 1834-1857, Sept. 2015.

[33] P. Setoodeh and S. Haykin, "Robust transmit power control for cognitive radio," Proc. IEEE, vol. 97, no. 5, pp. 915-939, May 2009.

[34] S. Boyd and L. Vandenberghe, Convex Optimization, Cambridge University Press, 2004.

[35] D. Xu and Q. Li, "Optimization of multiuser multichannel cognitive radio networks with wireless information and power transfer," WCSP, Yangzhou, 2016, pp. 1-5.

[36] A. Ben-Tal, L. E. Ghaoui, and A. Nemirovski, Robust Optimization, Princeton Series in Applied Mathematics, Princeton, NJ: Princeton Univ. Press, 2009.

[37] S. Parsaeefard and A. R. Sharafat, "Robust distributed power control in cognitive radio networks," IEEE Trans. Mobile Comput., vol. 12, no. 4 pp. 609-620, Apr. 2013.

[38] Y. Xu, Y. Hu, G. Li and H. Zhang, "Robust resource allocation for heterogeneous wireless network: A worst-case optimisation," IET Commun., vol. 12, no. 9, pp. 1064-1071, May 2018.

[39] Saeedeh Parsaeefard, Ahmad R Sharafat, "Worst-case robust distributed power allocation in shared unlicensed spectrum," arXiv preprint $\operatorname{arX}$ iv:1105.2989, 2011.

[40] D. G. Cacuci, Sensitivity and Uncertainty Analysis, Boca Raton, FL, USA: CRC Press, 2003

[41] S. Sun, W. Ni and Y. Zhu, "Robust power control in cognitive radio networks: a distributed way," ICC, Kyoto, 2011, pp. 1-6.

[42] A. Alizadeh and S. M. Sadough, "Power minimization in uni-directional relay networks with cognitive radio capabilities," IST, Tehran, 2010, pp. $18-22$.
[43] H. Q. Ngo, E. G. Larsson and T. L. Marzetta, "Energy and spectral efficiency of very large multiuser MIMO systems," IEEE Trans. Commun., vol. 61, no. 4, pp. 1436-1449, Apr. 2013. 